\title{
PICOT: A Multidomain Protein with Multiple Functions
}

\author{
Anna Keselman, Ranjan Nath Pulak, Keren Moyal, and Noah Isakov \\ The Shraga Segal Department of Microbiology and Immunology, Faculty of Health Sciences and the Cancer Research Center, \\ Ben-Gurion University of the Negev, P.O. Box 653, Beer-Sheva 84105, Israel \\ Correspondence should be addressed to Noah Isakov, noah@bgu.ac.il
}

Received 3 August 2011; Accepted 18 August 2011

Academic Editors: S.-I. Fujii, D. L. Jankovic, and P. Kisielow

Copyright ( $) 2011$ Anna Keselman et al. This is an open access article distributed under the Creative Commons Attribution License, which permits unrestricted use, distribution, and reproduction in any medium, provided the original work is properly cited.

\begin{abstract}
The PICOT protein possesses three highly conserved regions that include an aminoterminal thioredoxin-like homology domain and a tandem repeat of a carboxyterminal PICOT homology domain with an overall conformation that resembles a glutaredoxin homology domain. In contrast to the classical dithiol thioredoxins and glutaredoxins, PICOT possesses a single cysteine residue in each of its three domains and is therefore distinct from the classical thioredoxin and glutaredoxin redox enzymes. Recent studies demonstrated that PICOT is a prerequisite for mouse embryogenesis and participates in several independent biological systems in the adult. This paper examines advances made over the past few years in understanding the role of PICOT in various biological systems.
\end{abstract}

\section{Introduction}

The PICOT protein was discovered in studies of $\mathrm{T}$ cells aimed to elucidate the role of the protein kinase $\mathrm{C}$ theta $(\mathrm{PKC} \theta)$ isoform in the T-cell antigen receptor- (TCR-) linked signal transduction pathways. PKC $\theta$ is a member of the $\mathrm{Ca}^{2+}$-independent PKC subfamily of Ser/Thr kinases which is expressed predominantly in T-cells, platelets, and smooth muscle [1-5]. PKC $\theta$ plays a nonredundant role in T-cell activation and is the only $\mathrm{PKC}$ isoform that translocates to the plasma membrane of activated $\mathrm{T}$ cells upon interaction of their TCR with an MHC-bound peptide antigen on the surface of antigen-presenting cells (APCs) [6-8]. The T-cellAPC contact area undergoes rapid changes in protein content, leading to formation of a spatiotemporal structure, known as the immunological synapse (IS). PKC $\theta$ recruits to the center of the IS, where it interacts with and regulates the activity of selected effector molecules. As a result, these molecules mediate selected biochemical events required for signal transduction downstream of the activated TCR [9-11].

Under specific cellular conditions, PKC enzymes interact with various proteins that can regulate the conformation, activity, and subcellular location of the bound PKC [12-18]. In a search for $\mathrm{PKC} \theta$-binding proteins which potentially regulate $\mathrm{PKC} \theta$ in $\mathrm{T}$ cells, Witte et al. have utilized the yeast two-hybrid system in a search for PKC $\theta$-binding partners in a cDNA library of human Jurkat T lymphocytes [19]. These studies, performed in the year 2000, have led to the discovery of a new gene that was termed PICOT (PKC-interacting cousin of thioredoxin) based on its partial homology to the thioredoxin enzyme.

The existence of an aminoterminal thioredoxin-like homology domain in PICOT has led the HUGO Gene Nomenclature Committee (HGNC) to include PICOT, within the thioredoxin family, and rename it "thioredoxin-like 2" (gene symbol: TXNL-2). The current HGNC approved name and gene symbol for PICOT are "glutaredoxin 3" and "GLRX3", respectively, and its HGNC ID number is 15987 (http://www.genenames.org) [20]. PICOT has gained several different synonyms and alternative symbols, including HUSSY-22 [20], bA500G10.4, GLRX4, glutaredoxin 4, TXNL3, TXNL2, GRX3, GRX4, GLRX4, and FLJ11864.

\section{Structure of PICOT}

The PICOT protein is encoded by a unique gene, both in humans and mice, which has no other functional gene homologs in the entire genome. The PICOT gene was 
mapped to the human chromosome 10q26 and the mouse chromosome 7F5 (see Figure 1(a)). Human PICOT consists of 335 amino acids and a calculated molecular mass of $37,432 \mathrm{Da}$, whereas the mouse protein, which is highly homologous to the human protein, possesses two extra amino acids. Analysis of the genomic organization of the full-length coding sequence of the human PICOT gene indicated that it spans over 48,000 base pairs and includes 11 exons (see Figure $1(\mathrm{c})$ ) that are highly conserved between humans and mice.

The PICOT protein possesses an amino-terminal thioredoxin (Trx) homology domain (HD), followed by a tandem repeat of a PICOT-HD that resembles a glutaredoxin(Grx-) like HD and therefore bridges the thioredoxin and glutaredoxin families of enzymes. However, in contrast to thioredoxin, which possesses two critical cysteine residues in its catalytic site (within the motif, Cys-Gly-Pro-Cys) [21], PICOT possesses a single cysteine residue in its putative catalytic site (Ala-Pro-Gln-Cys) and is therefore enzymatically distinct from the thioredoxins. Furthermore, the two copies of the PICOT-HD, at the carboxyterminal of PICOT, are related in structure to glutaredoxins, which also consist of two critical cysteine residues in their catalytic site (within the conserved motif, Cys-Pro-Tyr-Cys) [22], while PICOT possesses just a single cysteine in its putative catalytic site (Cys-Gly-Phe-Ser).

A search of protein databases revealed that many proteins from different species possess one or more PICOT-HD as an integral part of the molecule. Within a large group of these proteins, the PICOT-HD was found at the protein's C-terminus, in close proximity to amino-terminal Trx- or Grx-like domains [23]. Based on the physical proximity of PICOT-HD and Trx- or Grx-HD in multiple proteins, it is possible that functional relationships exist between these domains. Furthermore, PICOT-HD, Trx-HD, and Grx$\mathrm{HD}$ are comprised of an overall similar globular topology [24]. Thus, each of the three domains possesses a canonical "thioredoxin fold domain," formed by a central mix of 4 or 5 strand $\beta$-sheets flanked by three or more $\alpha$-helices on either side of the $\beta$-strands $[25,26]$. A 3D structure of PICOT$\mathrm{HD}$ is presented in Figure 1(b), and additional information relevant to the amino acid sequence versus the topology of the PICOT-HD is included in [23]. The results imply that formation of the three domains during evolution originated from a single common ancestral gene.

A smaller group of PICOT-HD-containing proteins are built almost entirely of a PICOT-HD. It is possible therefore that PICOT-HD-containing proteins can perform biological activities independently of Trx or Grx. Alternatively, activity of these proteins may depend on their ability to dimerize or cooperate with other molecules, such as Trx or Grx.

\section{PICOT and Cell Growth Regulation}

PICOT is a ubiquitously expressed protein that has been found in most tested cell types, tissues, and organs. The wide-spread expression of PICOT was confirmed by several different methods, including Northern blot, RT-PCT, Western blot, and immunohistochemistry [19, 28-30]. Although
PICOT was originally identified as a $\mathrm{PKC} \theta$-binding protein, studies have shown that its expression is independent of $\mathrm{PKC} \theta$, since equal levels of PICOT proteins were observed in lymphoid organs of $\mathrm{PKC} \theta^{+/+}$and $\mathrm{PKC} \theta^{-/-}$mice [31].

PKC $\theta$ is essential for proper function of the TCR and for signal delivery from the activated receptor $[32,33]$. It plays an important role during the organization of the immunological synapse $[34,35]$ and is essential for proper responses of $\mathrm{T}$ cells to stimuli leading to proliferation and cytokine secretion $[32,33]$. In addition, PKC $\theta$ is required for $\mathrm{CD}^{+}{ }^{+} \mathrm{T}$-cell differentiation into selected T-cell subpopulations [36-38]. Initial studies demonstrated that PICOT might serve as a negative regulator of $\mathrm{PKC} \theta$ in $\mathrm{T}$ cells, since cotransfection of PICOT with a constitutively active PKC $\theta$ led to downregulation of PKC $\theta$-dependent activation of JNK and inhibition of the transcription factors AP- 1 and NF- $\kappa$ B [19].

Expression analyses of PICOT revealed higher protein levels in cell lines, compared to freshly isolated normal cells [29]. Because the majority of cell lines include rapidly growing transformed cells, the assumption that PICOT expression levels positively correlate with the cells' turnover was analyzed. In an experiment in which freshly isolated mouse spleen cells were in vitro cultured in the presence of a T-cell mitogen, the cells responded by increased cell multiplication, which directly correlated with an increase in the level of PICOT expression [31]. These data suggested that increased expression levels of PICOT promote cell growth or are the result of the rapid cell multiplication rate. However, other explanations could not be excluded, such as the effects of a high metabolic rate, time-dependent changes in oxygen tension, or other factors that exist in the in vitro culture and may affect PICOT expression.

Physiological activation of $\mathrm{T}$ lymphocytes is initiated following engagement of the T-cell antigen receptor (TCR) by the major histocompatibility complex- (MHC-) bound peptide antigen on the surface of antigen-presenting cells (APCs). This initial event leads to a rapid activation of TCRlinked protein tyrosine kinases (PTKs), which phosphorylate a plethora of effector molecules involved in signal transduction downstream of the activated receptor. Many of the early effects induced by the activated PTKs can be mimicked in vitro by cell treatment with reactive oxygen intermediates (ROS), such as hydrogen peroxide $\left(\mathrm{H}_{2} \mathrm{O}_{2}\right)$. Such compounds are normally produced in cells under certain activation conditions and serve as physiological regulators of various cellular functions [39-41], including lymphocyte activation [42-45]. Other cell types were also found to produce ROS in response to cytokines and growth factors [46-48]. One of the major sources for inducible ROS in leukocytes is the NADPH oxidase, which is activated by several mechanisms, including phosphorylation by PKC [49].

The fact that PICOT associates with PKC $\theta$ and possesses Trx- and Grx-like sequences suggests that it might be involved in redox-regulated biochemical processes. Indeed, Jurkat $\mathrm{T}$ cells treated with hydrogen peroxide responded by phosphorylation of PICOT on tyrosine residues, an effect that was sensitive to Src family PTK inhibitors [50]. Tyrosine phosphorylation of PICOT was also obtained following cell 


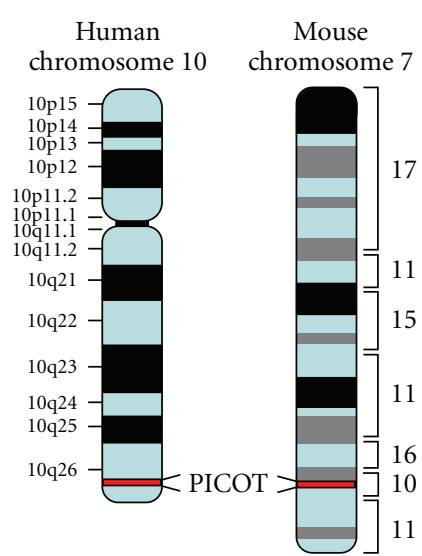

(a)

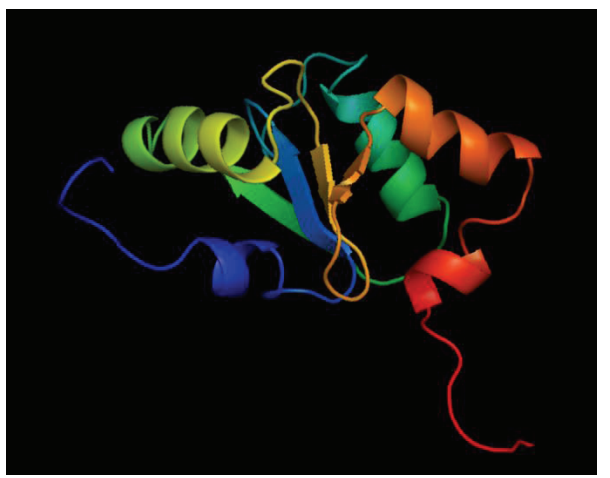

(b)

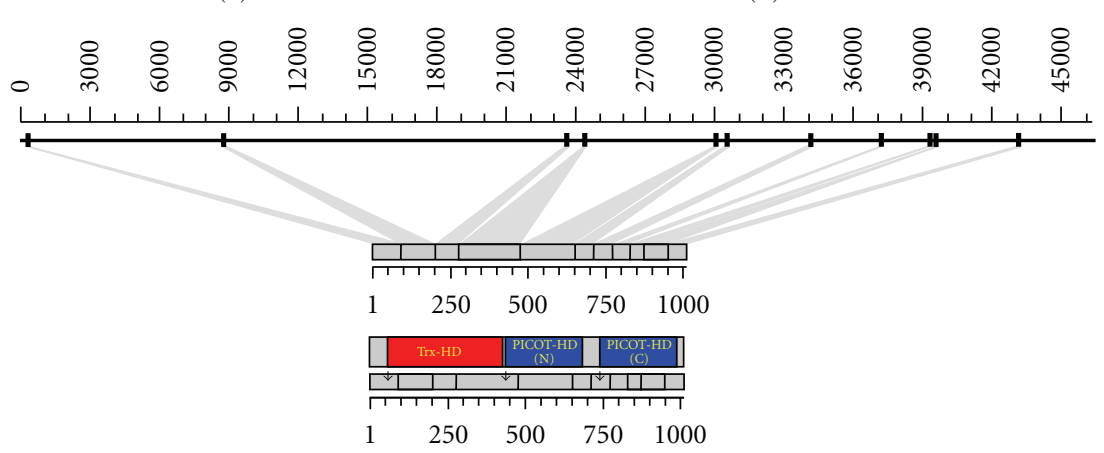

(c)

Figure 1: (a) The PICOT gene is located on human chromosome 10, on chromosomal region 10q26, and mouse chromosome 7, on chromosomal region 7 F5. (b) Three-dimensional structure of the PICOT homology domain. Solution structure of the PICOT$\mathrm{HD}(\mathrm{C})$ domain of the mouse PICOT protein was obtained by K. Miyamoto et al., using NMR analysis of a recombinant protein (DOI:10.2210/pdb1wik/pdb; http://www.genome.jp/dbget-bin/www_bget?pdb:1WIK) and drawn using the PyMOL Molecular Graphics System [27]. (c) Genomic organization of human PICOT. A schematic diagram of the genomic organization of the full-length coding sequence of the human PICOT gene. The gene is localized near the end of the long arm of chromosome 10, at 10q26, spans over 48,000 base pairs that include 11 exons and encodes a single protein of 335 amino acids.

transfection with a constitutively active Lck (Lck Y505F), suggesting that PICOT could serve as an Lck substrate. The results also imply that PICOT might play a role in cellactivation-dependent signaling pathways and/or responses to stress signals mediated by reactive oxygen intermediates.

A recent report by Kato et al. demonstrated the involvement of PICOT in the regulation of FceRI-mediated activation of mast cells [51]. In this study, overexpression of PICOT in the rat basophilic leukemia cell line, RBL-2H3, induced increased degranulation, concomitantly with activation of NF-AT and expression of IL- 4 and TNF $\alpha$, following FceRI crosslinking. In addition, analysis of fibroblasts derived from PICOT $^{-/-}$mouse embryos demonstrated an impaired cell growth that correlated with impaired cell cycle progression at the $\mathrm{G}_{2} / \mathrm{M}$ phase, and shRNA-mediated PICOT knockdown in HeLa cells led to a similar cell cycle defect during mitotic exit $[52,53]$. All these data support the important role PICOT plays in cell growth regulation via a mechanism which is presently unknown.

\section{PICOT and Cardiomyocytes}

Recent studies by Park and colleagues $[52,54,55]$ have established an important role for PICOT during hemodynamic stress-induced cardiac hypertrophy. In a search for potential regulators of myocardial mechanisms involved in responses to pathological stress, Jeong et al. have utilized a PCR-based subtractive RNA profiling of differentially regulated genes in the heart tissue of rats subjected to transverse aortic coarctation and control healthy rats [54]. PICOT was found to be among the genes preferentially expressed in hypertrophic hearts, and they substantiated this observation by Northern blot analysis, whereby PICOT expression in hypertrophied hearts increased $\sim 3$-fold compared to hearts from shamoperated rats. Overexpression of PICOT provided a partial protection to neonatal rat cardiomyocytes from hypertrophy induced by hypertrophic agonists, such as endothelin-1 (ET1) and phenylephrine (PE).

Considering the option that PICOT may also serve as an in vivo regulator of cardiac hypertrophy, Jeong et al. [54] 
have generated PICOT transgenic mice whereby PICOT is overexpressed under the regulation of the $\alpha$-myosin heavy chain (Mhc) gene promoter. They found that overexpression of PICOT in cardiac muscle cells significantly inhibited the pressure overload hypertrophy measured 2 weeks after transverse aortic banding. In addition, overexpressed PICOT increased the ventricular function and contractility of the cardiomyocytes.

In a complementary set of studies, Cha et al. [52] aimed at analyzing the sensitivity of PICOT-deficient mice to cardiac hypertrophy. Since the $\mathrm{PICOT}^{-/-}$mice they generated were embryonic lethal, Cha et al. utilized heterozygous PICOT $^{+/-}$mice that express $\sim 50 \%$ of the normal level of PICOT in the heart tissue. They found that decreased levels of PICOT in PICOT $^{+/-}$mice exacerbated the cardiac hypertrophy induced by pressure overload and decreased the in vitro contractility of cardiomyocytes derived from $\mathrm{PICOT}^{+/-}$newborn mice.

Using GST-PICOT in a pull-down assay, in conjunction with mass spectrometry, Jeong et al. have further searched for potential PICOT binding partners in the muscle tissue. They found that PICOT, via its carboxy terminus, directly interacts with the muscle LIM domain protein, MLP (also termed cysteine and glycine-rich protein 3; cardiac LIM protein (CLP); CRP3), a product of the CSRP3 gene [55], and a member of a family of cysteine-rich proteins that mediate proteinprotein interactions [56, 57]. MLP is a positive regulator of cell differentiation and myogenesis, and a component of the stretch sensor machinery in cardiomyocytes. MLP can interact with proteins, such as $\alpha$-actinin, zyxin, and calcineurin that are found at the Z-disc of the sarcomere [5860].

Immunofluorescence staining of tissue sections of adult mouse heart demonstrated that PICOT binds to and colocalizes with MLP at the Z-disc [55]. An additional protein that is linked to the Z-disc via MLP is the $\mathrm{Ca}^{2+}$-dependent Ser/Thr phosphatase, calcineurin [61]. Binding of calcineurin to the Z-disc allows it to dephosphorylate the cytoplasmic transcription factor, NF-AT, which can then translocate to the nucleus and initiate the transcription of NF-AT-regulated genes $[58,62]$.

PICOT association with MLP was found to compete with MLP binding to calcineurin, causing a dose-dependent displacement of calcineurin from the Z-disc. As a consequence, pressure-overload-mediated calcineurin activation was abrogated, thereby inhibiting the dephosphorylation and activation of NF-AT and the activation of NF-AT-regulated gene transcription. It is therefore suggested that the MLPcalcineurin-NF-AT signaling pathway in cardiomyocytes can be downregulated by PICOT that disrupts the MLPcalcineurin interaction, thereby preventing cardiac hypertrophy.

\section{PICOT and Cancer}

As mentioned earlier, transformed cell lines express higher levels of PICOT compared to normal cells of the same histological origin. This observation and the findings that
PICOT participates in signal transduction in various cell types raised the possibility that PICOT might have a role in cell growth regulation. To test whether the increased expression of PICOT in the in vitro growing transformed cells reflects the cancerous nature of these cells, or perhaps the in vitro conditions, PICOT expression levels were tested in in vivo growing tumors cells and compared to those of normal cells from the same histological origin.

Immunohistochemical staining of lymph nodes from Hodgkin's lymphoma patients revealed that the expression levels of PICOT in Hodgkin's lymphoma and Reed Sternberg cells in situ, were significantly higher than those of the surrounding normal lymphocytes within the same tissue biopsy [31].

RT-PCR analysis of PICOT expression in different types of human cancers demonstrated a 55.3-fold and 50.2-fold increase in PICOT levels in lung and colon cancer, respectively [28]. Furthermore, the increase in PICOT mRNA levels in the cancerous tissues was significantly higher than the increase observed in the levels of other glutaredoxin, thioredoxin, and peroxiredoxin family members. Western blot analysis confirmed the significant increase in PICOT protein levels in the lung and colon cancers. The extent of increase of PICOT protein and mRNA levels was not always in direct correlation, suggesting that expression of the PICOT protein is regulated by both translational and posttranslational mechanisms.

In a recent study by $\mathrm{Qu}$ and colleagues [63], the authors used the Oncomine database to compare PICOT expression levels in different normal and cancer tissues and found that PICOT is upregulated in a range of human cancer tissues, including breast, colon, lung, gastric, bladder, and cervical cancer. Immunoblot analysis and immunohistochemical staining confirmed that breast cancer cell lines and tissues express higher levels of PICOT. shRNA-mediated knockdown of PICOT in human breast cancer cell lines increased ROS levels and decreased NF- $\kappa$ B activity, concomitantly with inhibition of the cells growth rate, survival, and invasiveness. Transplantation of these cells to immune-deprived mice demonstrated that knockdown of PICOT inhibited tumorigenesis and metastasis of these cells. In addition, the expression levels of PICOT in primary human breast cancer were found to correlate with the increased frequency of metastasis formed in the lung and brain, and the overall decreased survival.

\section{PICOT and Embryogenesis}

Comparative analysis of protein profiles expressed at different stages of embryonic development is a useful tool for the identification of molecules involved in specific developmental processes. To determine protein profile changes during embryogenesis, Greene et al. [64] utilized an immobilized $\mathrm{pH}$ gradient-based two-dimensional electrophoresis and identified differentially expressed protein spots by mass spectrometry. Analysis of proteins expressed on days 8.5 (E8.5)-10.5 of embryogenesis, the time of formation of the neural tube, revealed a number of changes in protein 
patterns at successive embryonic days. PICOT was almost undetectable at E8.5 but was dramatically upregulated at E9.5, suggesting a role for PICOT in biological processes that occur in the developing embryo at midgestation. To further analyze potential roles for PICOT during mouse embryogenesis, Cha et al. have utilized a gene trap approach to prepare PICOT-deficient mice [52]. They found that ablation of the PICOT gene led to embryonic lethality around E12.5 to E14.5, indicating that PICOT is sine qua non for the ongoing development of the embryo at the postE12.5-E14.5 stage. Similar results were obtained in a different line of PICOT $^{-1-}$ mice, produced independently by Cheng and his colleagues [53]. The majority of PICOT $^{-1-}$ embryos appeared to be morphologically normal, but smaller in size compared to the heterozygous or wild-type embryos. Some E12.5 PICOT $^{-/-}$embryos displayed growth defects, such as open anterior neural tubes and pericardial effusion [53]. $\mathrm{PICOT}^{+/-}$heterozygous mice were viable with no apparent morphological defects and with PICOT protein levels that are $\sim 50 \%$ of those found in wild-type mice $[52,53]$. These two studies indicate that PICOT is critical for mouse embryonic development.

A study by Saito et al. [65] demonstrated that PICOT can interact with CIAPIN-1, also termed anamorsin or "cytokine-induced apoptosis inhibitor 1," a cell-death-defying factor and a cytokine-induced inhibitor of apoptosis [66]. CIAPIN-1 shares some general characteristics with PICOT, and its knockdown also results in embryonic lethality in late gestation [67]. In addition, CIAPIN-1 is expressed in several types of solid and hematopoietic human cancers, and its expression levels correlate with the tumor aggressiveness. It has therefore been suggested that CIAPIN- 1 and PICOT may act cooperatively during embryogenesis [65].

\section{Conclusions}

PICOT is an evolutionary conserved and ubiquitously expressed protein that is critical for mouse embryonic development. It also plays an important role in postembryonic growth and heart function, particularly under oxidative stress conditions leading to cardiac hypertrophy. An increase in PICOT expression levels in cardiac tissue is apparently required for coping with extreme changes in free oxygen radicals. Such a mechanism can provide a logical explanation for the findings showing that artificial overexpression of PICOT inhibits the pressure overload hypertrophy of the heart following transverse aortic banding and increases the ventricular function and contractility of cardiomyocytes. Increased expression of PICOT in response to stimuli that induce an oxidative stress was demonstrated in vivo in heart tissue [54] and in vitro, in mitogen-treated T lymphocytes [31], as well as in mouse myoblast cells exogenously treated with oxidizing agents [53].

Human PICOT has been characterized as an iron-sulfur (Fe/S) protein [68], similar to the PICOT homolog in yeast, Grx3, which was shown to play a crucial role in intracellular iron trafficking and sensing [69]. Iron-sulfur proteins are ubiquitously expressed and participate in diverse biochemical functions in virtually every living cell. They consist of two or more iron atoms bridged by sulfur ligands and perform multiple tasks, including respiration, oxidationreduction reactions, heme biosynthesis, iron homeostasis, and regulation of gene expression.

The fact that PICOT can physically interact with several different functionally nonrelated proteins and possesses highly conserved sequences with resemblance to both thioredoxins and glutaredoxins suggests that PICOT may be involved in several different biological tasks, which may also differ among distinct cell types. Understanding the mechanism of action of PICOT may have significant implications on a range of clinical situations, including myocardial infarction and cancer diseases, which evoke inflammatory responses triggered by the local hypoxia that could impact on the disease progression.

\section{Abbreviations}

$\begin{array}{ll}\text { PICOT,: } & \text { PKC-interacting cousin of thioredoxin } \\ \text { Glutaredoxin 3: } & \text { GLRX3 } \\ \text { TXNL2: } & \text { Thioredoxin-like protein } 2 \\ \text { PKC: } & \text { Protein kinase C. }\end{array}$

\section{Acknowledgments}

Work in our laboratory is funded in part by the USA-Israel Binational Science Foundation, the Israel Science Foundation administered by the Israel Academy of Science and Humanities, and a donation by Linda Osofsky. N. Isakov holds the Joseph H. Krupp Chair in Cancer Immunobiology.

\section{References}

[1] G. Baier, D. Telford, L. Giampa et al., "Molecular cloning and characterization of $\mathrm{PKC} \theta$, a novel member of the protein kinase $\mathrm{C}(\mathrm{PKC})$ gene family expressed predominantly in hematopoietic cells," The Journal of Biological Chemistry, vol. 268, no. 7, pp. 4997-5004, 1993.

[2] J. D. Chang, Y. Xu, M. K. Raychowdhury, and J. A. Ware, "Molecular cloning and expression of a cDNA encoding a novel isoenzyme of protein kinase $\mathrm{C}$ (nPKC). A new member of the nPKC family expressed in skeletal muscle, megakaryoblastic cells, and platelets," The Journal of Biological Chemistry, vol. 268, no. 19, pp. 14208-14214, 1993.

[3] L. Hilgenberg and K. Miles, "Developmental regulation of a protein kinase $\mathrm{C}$ isoform localized in the neuromuscular junction," Journal of Cell Science, vol. 108, part 1, pp. 51-61, 1995.

[4] N. Meller, Y. Elitzur, and N. Isakov, "Protein kinase C- $\theta$ $(\mathrm{PKC} \theta)$ distribution analysis in hematopoietic cells: proliferating T cells exhibit high proportions of $\mathrm{PKC} \theta$ in the particulate fraction," Cellular Immunology, vol. 193, no. 2, pp. 185-193, 1999.

[5] S. Cohen, A. Braiman, G. Shubinsky, A. Ohayon, A. Altman, and $\mathrm{N}$. Isakov, "PKC $\theta$ is required for hemostasis and positive regulation of thrombin-induced platelet aggregation and $\alpha$-granule secretion," Biochemical and Biophysical Research Communications, vol. 385, no. 1, pp. 22-27, 2009.

[6] C. R. F. Monks, B. A. Freiberg, H. Kupfer, N. Sciaky, and A. Kupfer, "Three-dimensional segregation of supramolecular 
activation clusters in T cells," Nature, vol. 395, no. 6697, pp. 82-86, 1998.

[7] C. R. F. Monks, H. Kupfer, I. Tamir, A. Barlow, and A. Kupfer, "Selective modulation of protein kinase C- $\theta$ during T-cell activation," Nature, vol. 385, no. 6611, pp. 83-86, 1997.

[8] A. Altman, N. Isakov, and G. Baier, "Protein kinase C $\theta$ : a new essential superstar on the T-cell stage," Immunology Today, vol. 21, no. 11, pp. 567-573, 2000.

[9] Y. Sasahara, R. Rachid, M. J. Byrne et al., "Mechanism of recruitment of WASP to the immunological synapse and of its activation following TCR ligation," Molecular Cell, vol. 10, no. 6, pp. 1269-1281, 2002.

[10] T. N. Sims, T. J. Soos, H. S. Xenias et al., "Opposing effects of PKC $\theta$ and WASp on symmetry breaking and relocation of the immunological synapse," Cell, vol. 129, no. 4, pp. 773-785, 2007.

[11] K. Bi, Y. Tanaka, N. Coudronniere et al., "Antigen-induced translocation of PKC- $\theta$ to membrane rafts is required for $\mathrm{T}$ cell activation," Nature Immunology, vol. 2, no. 6, pp. 556-563, 2001.

[12] D. Mochly-Rosen, H. Khaner, and J. Lopez, "Identification of intracellular receptor proteins for activated protein kinase C," Proceedings of the National Academy of Sciences of the United States of America, vol. 88, no. 9, pp. 3997-4000, 1991.

[13] C. Chapline, K. Ramsay, T. Klauck, and S. Jaken, "Interaction cloning of protein kinase C substrates," The Journal of Biological Chemistry, vol. 268, no. 10, pp. 6858-6861, 1993.

[14] D. Mochly-Rosen, "Localization of protein kinases by anchoring proteins: a theme in signal transduction," Science, vol. 268, no. 5208, pp. 247-251, 1995.

[15] N. Meller, Y. C. Liu, T. L. Collins et al., "Direct interaction between protein kinase $\mathrm{C} \theta$ (PKC $\theta)$ and $14-3-3 \tau$ in T cells: $14-$ 3-3 overexpression results in inhibition of $\mathrm{PKC} \theta$ translocation and function," Molecular and Cellular Biology, vol. 16, no. 10, pp. 5782-5791, 1996.

[16] G. C. Blobe, D. S. Stribling, D. Fabbro, S. Stabel, and Y. A. Hannun, "Protein kinase C $\beta$ II specifically binds to and is activated by F-actin," The Journal of Biological Chemistry, vol. 271, no. 26, pp. 15823-15830, 1996.

[17] C. Chapline, J. Cottom, H. Tobin, J. Hulmes, J. Crabb, and S. Jaken, "A major, transformation-sensitive PKC-binding protein is also a PKC substrate involved in cytoskeletal remodeling," The Journal of Biological Chemistry, vol. 273, no. 31, pp. 19482-19489, 1998.

[18] T. M. Klauck, M. C. Faux, K. Labudda, L. K. Langeberg, S. Jaken, and J. D. Scott, "Coordination of three signaling enzymes by AKAP79, a mammalian scaffold protein," Science, vol. 271, no. 5255, pp. 1589-1592, 1996.

[19] S. Witte, M. Villalba, K. Bi, Y. Liu, N. Isakov, and A. Altman, "Inhibition of the c-Jun N-terminal kinase/AP-1 and NF$\kappa \mathrm{B}$ pathways by PICOT, a novel protein kinase C-interacting protein with a thioredoxin homology domain," The Journal of Biological Chemistry, vol. 275, no. 3, pp. 1902-1909, 2000.

[20] F. Stanchi, E. Bertocco, S. Toppo et al., "Characterization of 16 novel human genes showing high similarity to yeast sequences," Yeast, vol. 18, no. 1, pp. 69-80, 2001.

[21] G. Powis, D. Mustacich, and A. Coon, "The role of the redox protein thioredoxin in cell growth and cancer," Free Radical Biology and Medicine, vol. 29, no. 3-4, pp. 312-322, 2000.

[22] N. Foloppe and L. Nilsson, "The glutaredoxin -C-P-Y-C- motif: influence of peripheral residues," Structure, vol. 12, no. 2, pp. 289-300, 2004.

[23] N. Isakov, S. Witte, and A. Altman, "PICOT-HD: a highly conserved protein domain that is often associated with thioredoxin and glutaredoxin modules," Trends in Biochemical Sciences, vol. 25, no. 11, pp. 537-539, 2000.

[24] E. Herrero and M. A. de la Torre-Ruiz, "Monothiol glutaredoxins: a common domain for multiple functions," Cellular and Molecular Life Sciences, vol. 64, no. 12, pp. 1518-1530, 2007.

[25] L. N. Kinch, D. Baker, and N. V. Grishin, "Deciphering a novel thioredoxin-like fold family," Proteins, vol. 52, no. 3, pp. 323331, 2003.

[26] Y. Qi and N. V. Grishin, "Structural classification of thioredoxin-like fold proteins," Proteins, vol. 58, no. 2, pp. 376388, 2005.

[27] W. L. Delano, The PyMOL Molecular Graphics System, DeLano Scientific, San Carlos, Calif, USA, 2002.

[28] M. K. Cha and I. H. Kim, "Preferential overexpression of glutaredoxin3 in human colon and lung carcinoma," Cancer Epidemiology, vol. 33, no. 3-4, pp. 281-287, 2009.

[29] A. Ohayon, Y. Babichev, M. Galperin, A. Altman, and N. Isakov, "Widespread expression of PICOT in mouse and human tissues with predominant localization to epithelium," Journal of Histochemistry and Cytochemistry, vol. 58, no. 9, pp. 799-806, 2010.

[30] J. R. Godoy, M. Funke, W. Ackermann et al., "Redox atlas of the mouse. Immunohistochemical detection of glutaredoxin-, peroxiredoxin-, and thioredoxin-family proteins in various tissues of the laboratory mouse," Biochimica et Biophysica Acta, vol. 1810, no. 1, pp. 2-92, 2011.

[31] A. Ohayon, Y. Babichev, R. Pasvolsky et al., "Hodgkin's lymphoma cells exhibit high expression levels of the PICOT protein," Journal of Immunotoxicology, vol. 7, no. 1, pp. 8-14, 2010.

[32] Z. Sun, C. W. Arendt, W. Ellmeier et al., "PKC- $\theta$ is required for TCR-induced NF- $\kappa \mathrm{B}$ activation in mature but not immature T lymphocytes," Nature, vol. 404, no. 6776, pp. 402-407, 2000.

[33] N. Isakov and A. Altman, "Protien kinase C $\theta$ in T cell activation," Annual Review of Immunology, vol. 20, pp. 761-794, 2002.

[34] S. K. Bromley, W. R. Burack, K. G. Johnsonn et al., "The immunological synapse," Annual Review of Immunology, vol. 19, pp. 375-396, 2001.

[35] C. W. Arendt, B. Albrecht, T. J. Soos, and D. R. Littman, "Protein kinase C- $\theta$ : signaling from the center of the T-cell synapse," Current Opinion in Immunology, vol. 14, no. 3, pp. 323330, 2002.

[36] B. J. Marsland, T. J. Soos, G. Späth, D. R. Littman, and M. Kopf, "Protein kinase C $\theta$ is critical for the development of in vivo T helper (Th)2 cell but not Th-1 cell responses," The Journal of Experimental Medicine, vol. 200, no. 2, pp. 181-189, 2004.

[37] S. Salek-Ardakani, T. So, B. S. Halteman, A. Altman, and M. Croft, "Differential regulation of Th2 and Th1 lung inflammatory responses by protein kinase C $\theta$," Journal of Immunology, vol. 173, no. 10, pp. 6440-6447, 2004.

[38] S. Salek-Ardakani, T. So, B. S. Halteman, A. Altman, and M. Croft, "Protein kinase C $\theta$ controls Th1 cells in experimental autoimmune encephalomyelitis," Journal of Immunology, vol. 175, no. 11, pp. 7635-7641, 2005.

[39] J. H. Shaw, L. Xiang, A. Shah, W. Yin, and P. G. Lloyd, "Placenta growth factor expression is regulated by hydrogen peroxide in vascular smooth muscle cells," The American Journal of Physiology, vol. 300, no. 2, pp. C349-C355, 2011.

[40] E. Veal and A. Day, "Hydrogen peroxide as a signaling molecule," Antioxidants \& Redox Signaling, vol. 15, no. 1, pp. 147$151,2011$. 
[41] G. Groeger, C. Quiney, and T. G. Cotter, "Hydrogen peroxide as a cell-survival signaling molecule," Antioxidants e Redox Signaling, vol. 11, no. 11, pp. 2655-2671, 2009.

[42] M. Monte, L. E. Davel, and E. Sacerdote de Lustig, "Hydrogen peroxide is involved in lymphocyte activation mechanisms to induce angiogenesis," European Journal of Cancer Part A, vol. 33, no. 4, pp. 676-682, 1997.

[43] L. F. Rosa, "Effect of adrenaline on lymphocyte metabolism and function, a mechanism involving cAMP and hydrogen peroxide," Cell Biochemistry and Function, vol. 15, no. 2, pp. 103-112, 1997.

[44] M. Reth, "Hydrogen peroxide as second messenger in lymphocyte activation," Nature Immunology, vol. 3, no. 12, pp. 1129 1134, 2002.

[45] J. Kwon, S. Devadas, and M. S. Williams, "T cell receptorstimulated generation of hydrogen peroxide inhibits MEKERK activation and lck serine phosphorylation," Free Radical Biology \& Medicine, vol. 35, no. 4, pp. 406-417, 2003.

[46] B. Meier, H. H. Radeke, S. Selle et al., "Human fibroblasts release reactive oxygen species in response to interleukin-1 or tumour necrosis factor- $\alpha$," The Biochemical Journal, vol. 263, no. 2, pp. 539-545, 1989.

[47] M. Ohba, M. Shibanuma, T. Kuroki, and K. Nose, "Production of hydrogen peroxide by transforming growth factor- $\beta 1$ and its involvement in induction of egr-1 in mouse osteoblastic cells," The Journal of Cell Biology, vol. 126, no. 4, pp. 10791088, 1994.

[48] M. J. Anasagasti, A. Alvarez, J. J. Martin, L. Mendoza, and F. Vidal-Vanaclocha, "Sinusoidal endothelium release of hydrogen peroxide enhances very late antigen-4-mediated melanoma cell adherence and tumor cytotoxicity during interleukin-1 promotion of hepatic melanoma metastasis in mice," Hepatology, vol. 25, no. 4, pp. 840-846, 1997.

[49] E. P. Reeves, L. V. Dekker, L. V. Forbes et al., "Direct interaction between $\mathrm{p} 47$ (phox) and protein kinase C: evidence for targeting of protein kinase C by p47(phox) in neutrophils," The Biochemical Journal, vol. 344, no. 3, pp. 859-866, 1999.

[50] Y. Babichev and N. Isakov, "Tyrosine phosphorylation of picot and its translocation to the nucleus in response of human $\mathrm{t}$ cells to oxidative stress," Advances in Experimental Medicine and Biology, vol. 495, pp. 41-45, 2001

[51] N. Kato, S. Motohashi, T. Okada, T. Ozawa, and K. Mashima, "PICOT, protein kinase $\mathrm{C}$ theta-interacting protein, is a novel regulator of FcepsilonRI-mediated mast cell activation," Cellular Immunology, vol. 251, pp. 62-67, 2008.

[52] H. Cha, J. M. Kim, J. G. Oh et al., "PICOT is a critical regulator of cardiac hypertrophy and cardiomyocyte contractility," Journal of Molecular and Cellular Cardiology, vol. 45, no. 6, pp. 796-803, 2008.

[53] N. H. Cheng, W. Zhang, W. Q. Chen et al., "A mammalian monothiol glutaredoxin, Grx3, is critical for cell cycle progression during embryogenesis," The FEBS Journal, vol. 278, no. 14, pp. 2525-2539, 2011.

[54] D. Jeong, H. Cha, E. Kim et al., "PICOT inhibits cardiac hypertrophy and enhances ventricular function and cardiomyocyte contractility," Circulation Research, vol. 99, no. 3, pp. 307-314, 2006.

[55] D. Jeong, J. M. Kim, H. Cha et al., "PICOT attenuates cardiac hypertrophy by disrupting calcineurin-NFAT signaling," Circulation Research, vol. 102, no. 6, pp. 711-719, 2008.

[56] G. V. Harrod, P. J. Kettunen, and A. K. Jowett, "Murine MLP: cloning and expression in the embryonic head," Journal of Craniofacial Genetics and Developmental Biology, vol. 16, no. 2, pp. 65-73, 1996.
[57] B. Buyandelger, K. E. Ng, S. Miocic et al., "MLP (muscle LIM protein) as a stress sensor in the heart," Pflugers ArchivEuropean Journal of Physiology, vol. 462, no. 1, pp. 135-142, 2011.

[58] J. Heineke, H. Ruetten, C. Willenbockel et al., "Attenuation of cardiac remodeling after myocardial infarction by muscle LIM protein-calcineurin signaling at the sarcomeric Z-disc," Proceedings of the National Academy of Sciences of the United States of America, vol. 102, no. 5, pp. 1655-1660, 2005.

[59] K. Gehmlich, C. Geier, K. J. Osterziel, P. F. M. Van der Ven, and D. O. Fürst, "Decreased interactions of mutant muscle LIM protein (MLP) with N-RAP and $\alpha$-actinin and their implication for hypertrophic cardiomyopathy," Cell and Tissue Research, vol. 317, no. 2, pp. 129-136, 2004.

[60] B. Mohapatra, S. Jimenez, J. H. Lin et al., "Mutations in the muscle LIM protein and $\alpha$-actinin-2 genes in dilated cardiomyopathy and endocardial fibroelastosis," Molecular Genetics and Metabolism, vol. 80, no. 1-2, pp. 207-215, 2003.

[61] D. Frank and N. Frey, "Cardiac Z-disc signaling network," The Journal of Biological Chemistry, vol. 286, no. 12, pp. 98979904, 2011.

[62] J. D. Molkentin, "Calcineurin-NFAT signaling regulates the cardiac hypertrophic response in coordination with the MAPKs," Cardiovascular Research, vol. 63, no. 3, pp. 467-475, 2004.

[63] Y. Qu, J. Wang, P. S. Ray et al., “Thioredoxin-like 2 regulates human cancer cell growth and metastasis via redox homeostasis and NF- $\kappa \mathrm{B}$ signaling," The Journal of Clinical Investigation, vol. 121, no. 1, pp. 212-225, 2011.

[64] N. D. E. Greene, K. Y. Leung, R. Wait, S. Begum, M. J. Dunn, and A. J. Copp, "Differential protein expression at the stage of neural tube closure in the mouse embryo," The Journal of Biological Chemistry, vol. 277, no. 44, pp. 41645-41651, 2002.

[65] Y. Saito, H. Shibayama, H. Tanaka, A. Tanimura, I. Matsumura, and Y. Kanakura, "PICOT is a molecule which binds to anamorsin," Biochemical and Biophysical Research Communications, vol. 408, no. 2, pp. 329-333, 2011.

[66] H. Shibayama, E. Takai, I. Matsumura et al., "Identification of a cytokine-induced antiapoptotic molecule anamorsin essential for definitive hematopoiesis," The Journal of Experimental Medicine, vol. 199, no. 4, pp. 581-592, 2004.

[67] Y. Saito, H. Shibayama, H. Tanaka, A. Tanimura, and Y. Kanakura, "A cell-death-defying factor, anamorsin mediates cell growth through inactivation of PKC and p38MAPK," Biochemical and Biophysical Research Communications, vol. 405, no. 2, pp. 303-307, 2011.

[68] P. Haunhorst, C. Berndt, S. Eitner, J. R. Godoy, and C. H. Lillig, "Characterization of the human monothiol glutaredoxin 3 (PICOT) as iron-sulfur protein," Biochemical and Biophysical Research Communications, vol. 394, no. 2, pp. 372-376, 2010.

[69] U. Mühlenhoff, S. Molik, J. R. Godoy et al., "Cytosolic monothiol glutaredoxins function in intracellular iron sensing and trafficking via their bound iron-sulfur cluster," Cell Metabolism, vol. 12, no. 4, pp. 373-385, 2010. 


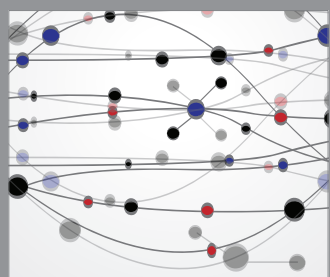

The Scientific World Journal
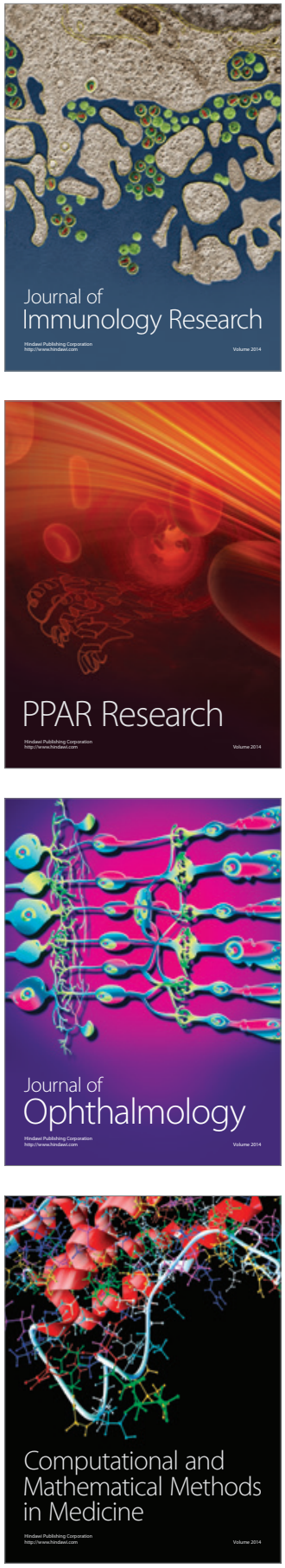

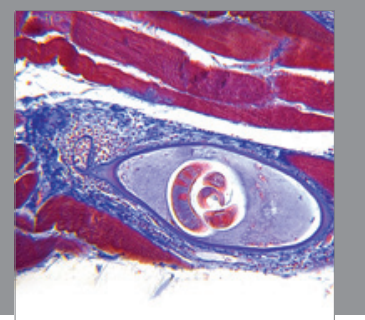

Gastroenterology

Research and Practice
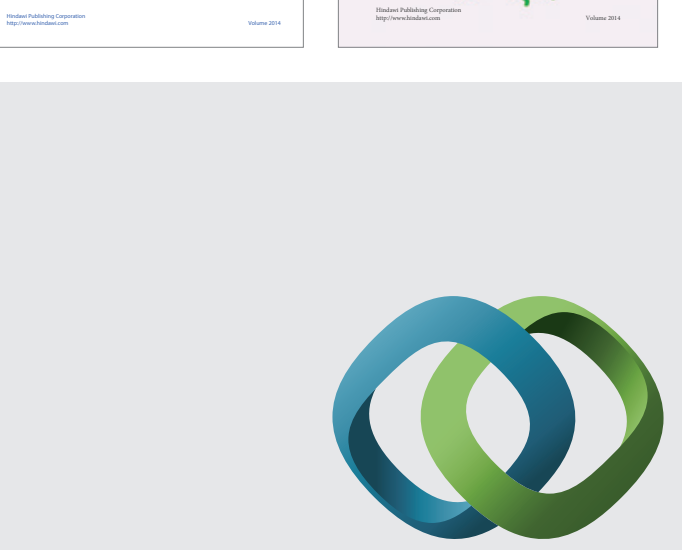

\section{Hindawi}

Submit your manuscripts at

http://www.hindawi.com
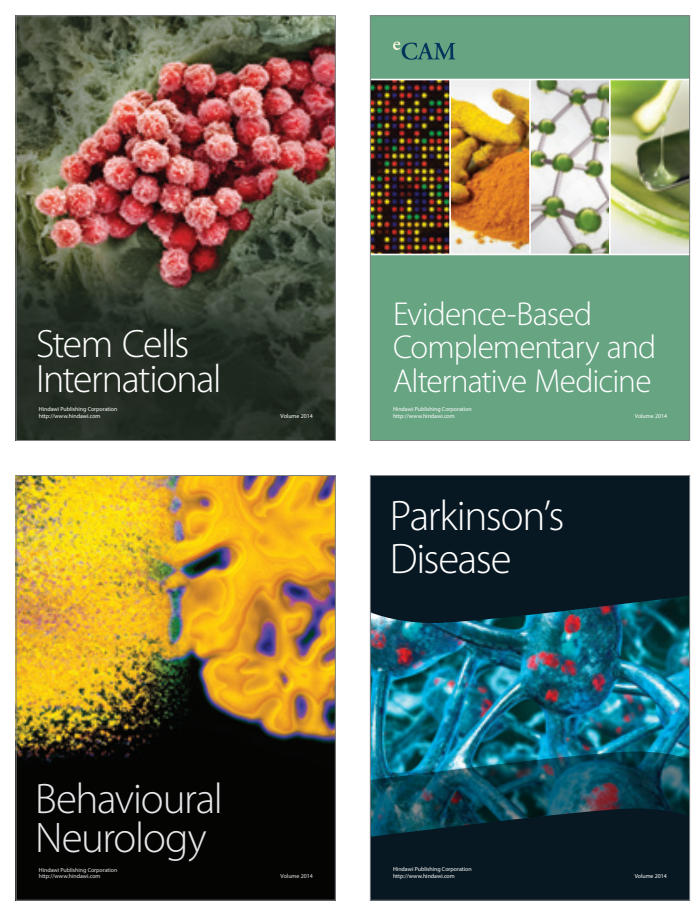

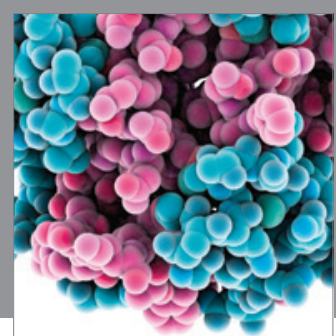

Journal of
Diabetes Research

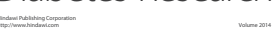

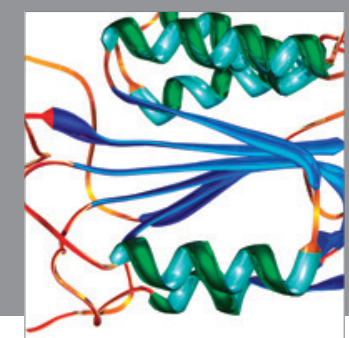

Disease Markers
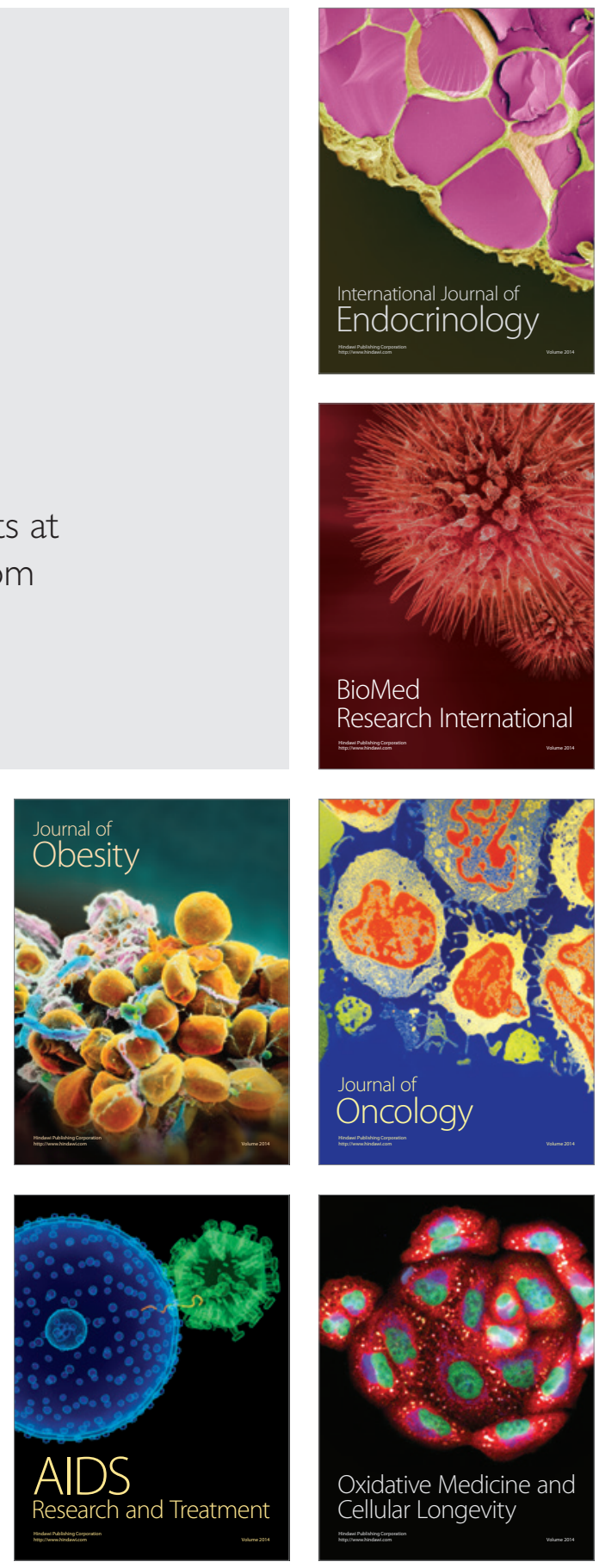